\title{
Determination of atmospheric column condensate using active and passive remote sensing technology
}

\author{
Huige Di ${ }^{1}$, Yun Yuan ${ }^{1}$, Qing Yan ${ }^{1}$, Wenhui Xin ${ }^{1}$, Shichun $\mathrm{Li}^{1}$, Jun Wang ${ }^{1}$, Yufeng Wang ${ }^{1}$, Lei \\ Zhang $^{2}$, Dengxin Hua ${ }^{1 *}$
}

$5 \quad{ }^{1}$ School of Mechanical and Precision Instrument Engineering, Xi'an University of Technology, Xi'an 710048, China ${ }^{2}$ College of Atmospheric Sciences, Lanzhou University, Lanzhou 730000, China

Correspondence to: Dengxin Hua (dengxinhua@xaut.edu.cn)

\begin{abstract}
To further exploit atmospheres cloud-water resources (CWR), it is necessary to correctly evaluate the amount of CWR in an area. CWR are hydrometeors that have not participated in precipitation formation at the surface and are 10 suspended in the atmosphere to be exploited to maximize possible precipitation in the atmosphere (Zhou, Y., et al. (2020)). CWR includes three items: the existing hydrometeors at a time, the influx of atmospheric hydrometeors along the boundaries of the study area, and the mass of hydrometeors converted from water vapor through condensation or desublimation, defined as condensate. Condensate is the most important part of CWR. At present, there is a lack of effective observation methods for atmospheric column condensates, so the direct observation data of CWR are insufficient. The method for detecting

15 atmospheric column condensate in the atmosphere is proposed and presented. The formation of condensate is closely related to atmospheric meteorological parameters (e.g., temperature and vertical airflow velocity). For stratiform clouds, the amount of atmospheric column condensate can be calculated by the saturated water vapor density and the ascending velocity at the cloud base and top. Active and passive remote sensing technology are applied to detect the mass of atmospheric column condensate. Combining millimetre-wave radar (MWR), lidar and microwave radiometers can well observe the vertical

20 velocity and temperature at the cloud boundary. The detailed detection scheme and data calculation method are presented, and the presented method can realize the deduction of atmospheric column condensate. A case of cloud layer change before precipitation was monitored, and atmospheric column condensate was deduced and obtained. This is the first application, to our knowledge, to operate observations of atmospheric column condensates, which is significant for research on the hydrologic cycle and the assessment of cloud water resources.
\end{abstract}

1 Introduction

Water is a renewable but finite natural resource. Although three-quarters of the world's surface is covered with water, the freshwater resources available for human uses are very limited. Its availability varies significantly over space and time. With the increasing water consumption caused by the human population, freshwater will become an increasingly scarce resource in the future (Yoshiaki et al., 2009). In principle, freshwater resources all come from precipitation. Precipitation, produced 
30 by the atmospheric water cycle, is the main source of river runoff and shallow underground freshwater that can be directly used by humans (Zhou et al., 2020). The atmospheric water cycle is one of the branches of the water cycle (Su et al., 2014). It arises from evaporation of the oceans, lakes and rivers, which is then transported within the atmosphere and condenses into clouds; finally, it precipitates to the ground before evaporating again (Yao et al., 2020). Atmospheric water includes water vapor and hydrometeors (also termed cloud water in both liquid and solid phases). The atmospheric water vapor resources on Earth are very rich, while water vapor cannot directly precipitate, and only the water substance that has experienced the second phase change and turned into the liquid/solid phase may form rainfall, which is defined as CWR (Zhou et al., 2020). CWR refers to hydrometeors that participate in atmospheric water change in a certain area and time period but have not formed ground precipitation and thus can be utilized/exploited (Jalihal et al., 2019). The formation of hydrometers is affected by dynamic and thermodynamic processes in the atmosphere, which is playing an important role in

40 the global water cycle.

Arid and semiarid areas cover more than half of China's land area. Water shortages are serious in China. It is an urgent task to rationally develop CWRs in the air. Approximately 14\% 18\% of water vapor in the air can condense into cloud water and precipitate to the ground, so there is still great potential for the development of CWR. It is of great strategic significance to carry out comprehensive research on the development and application of atmospheric water resources and realize the optimal

45 allocation of CWR to alleviate the current situation of uneven drought and flooding in China. The first step of exploiting CWR is to correctly evaluate CWR in an area, that is, the potential precipitation of the cloud system. At present, the evaluation of CWR is mainly based on reanalysis data or mesoscale numerical simulations (Zhou et al., 2011). Due to the lack of observation data of CWR, the evaluation results of CWR using numerical simulations have not been verified, which greatly affects the effectiveness and economic applicability of weather modification. It is urgent to develop instruments and 50 methods that can observe CWR.

The CWR is closely related to various macro- and microparameters of clouds and multiple atmospheric parameters, and they mainly depend on the distribution of the water vapor supply and updraft (Jalihal et al., 2019). Researchers have carried out many observations on the macro- and microphysical characteristics of clouds through ground-based, aircraft and satellite equipment (Williams et al., 2016; Chetankumar Jalihal, et al. 2019; Liu et al., 2013; Si et al., 2019). Meteorological satellites

55 can provide a more accurate assessment of global cloud distribution. The cloud products provided by meteorological satellites mainly include cloud cover, cloud water paths, cloud optical thickness, and more. In previous studies, the cloud water path was generally regarded as the CWR. According to the definition of CWR proposed by Zhou et al. (Zhou et al.,2020), the state quantity of hydrometeors is only a small part of the CWR (Zhou et al., 2011). The mass of hydrometeors converted from water vapor caused by updraft through condensation or desublimation is an important part. However, there

60 are no methods or means for the observation of cloud water caused by updrafts.

Supported by the National Natural Science Foundation of China's major research instrument development project, Xi'an University of Technology and Lanzhou University carried out a study on cloud potential precipitation assessment and observation technology, focusing on remote sensing detection and atmospheric column condensation. Based on the 
formation mechanism of condensate in the atmosphere, this paper proposes a method for detecting atmospheric column condensate. Remote sensing technologies combining lidar, MWR and microwave radiometry were used for the determination of atmospheric column condensation, and experimental observations were carried out.

\section{CWR}

According to the atmospheric water cycle process, only the water condensate in the cloud can grow into raindrops to form precipitation, and potential precipitation refers to the total mass of cloud water in a certain region and period, including the amount of cloud water in the region at the beginning of a period, the amount of cloud water input horizontally in the period and the amount of condensed cloud water vertically. Fig. 1 shows a schematic diagram of CWR.

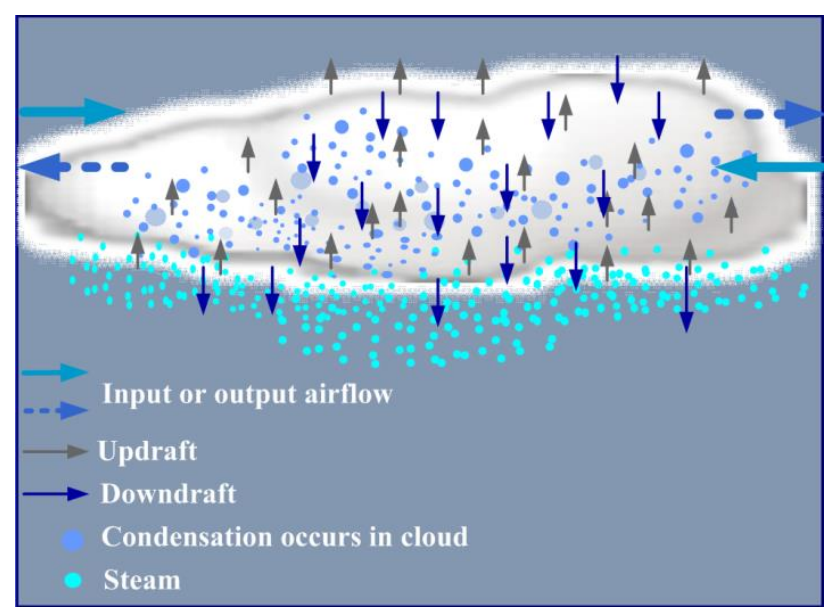

Figure 1: Schematic diagram of CWR

Based on the atmospheric water substance budget and water mass conservation, for an atmospheric column from the ground

75 to the top of the atmosphere, the budget equation of atmospheric hydrometeors can be expressed by the following equation (Zhou et al., 2020).

$$
M_{\mathrm{h} 1}+Q_{\mathrm{hi}}+C_{\mathrm{vh}}=M_{\mathrm{h} 2}+Q_{\mathrm{ho}}+C_{\mathrm{hv}}+P_{\mathrm{S}}=\mathrm{GM}_{\mathrm{h}} \text {, }
$$

The left side of the equation is the income item of the atmospheric hydrometeors, and the right side is the expenditure item. $M_{\mathrm{h} 1}$ and $M_{\mathrm{h} 2}$ are the masses of hydrometeors at time moments $t_{1}$ and $t_{2}$. $Q_{\mathrm{hi}}$ and $Q_{\mathrm{ho}}$ are the influx and outflux of atmospheric

80 hydrometeors along the boundaries of the study area. $C_{\mathrm{vh}}$ is the mass of hydrometeors converted from water vapor through condensation or sublimation, and $C_{\mathrm{hv}}$ is the mass content of water vapor converted from hydrometeors through evaporation or sublimation. $P_{\mathrm{S}}$ is the surface precipitation. $\mathrm{GM}_{\mathrm{h}}$ is the gross mass of atmospheric hydrometeors, representing the total amount of hydrometeors over the time period $t_{2}-t_{1}$ within the study region.

$M_{\mathrm{h} 1}$ at time $t_{1}$, that is, cloud liquid water content (CLW) and ice water content (IWC), can be observed by remote sensing

85 instruments, such as cloud radar, microwave radiometer and imaging spectroradiometer (Hengchi et al., 2003). Many spaceborne observations of the column-integrated cloud liquid water path (LWP) exist. After many years of development, 
the inversion algorithm of CLW using satellite remote sensing data is now mature (Jussi et al. 2016). $Q_{\mathrm{hi}}$ and $Q_{\mathrm{ho}}$ are related to the horizontal wind speed and cloud water content on the boundary, which can be measured by wind profiler radar and cloud radar. In addition to the existing cloud water and horizontal inputs, the mass content of water vapor converted from

90 hydrometeors through evaporation or sublimation accounts for a large proportion of cloud water resources. Updrafts and sufficient water vapor are necessary for cloud formation. To a certain extent, the condensate produced by the updraft is the main indicator of whether the cloud system can produce rainfall. For synoptic scale and mesoscale precipitation processes, the mass of the condensate is 100 times and 10 times larger than the cloud water input from the side boundary, respectively.

\section{Atmospheric column condensate}

95 The cloud is a saturated wet system with liquid water (or ice particles), which is a binary multiphase system. When the water vapor under the cloud is transported to the upper space (cloud layer) by updraft and the water vapor reaches saturation in cooler air, the excess water vapor will condense and form water condensate (Gu et al., 2006). The condensate produced by updrafts in cloud systems cannot be directly observed and quantified as in the above CLW.

Most of the important thermal processes in the atmosphere, especially the vertical motion of air, can be considered adiabatic;

100 therefore, atmospheric column condensate can be estimated by the saturated water vapor density and the ascending velocity at the cloud base. Of course, not all the water vapor entering cloud layer from the base can be transformed into condensate. A part of the water vapor will escape if there is an updraft at the cloud top. It is also possible that some water vapor does not condense and exists in the cloud as supersaturated water vapor. However, it is certain that the more water vapor enters the cloud, the more condensate may be generated. If we can obtain the saturated water vapor density and vertical air velocity at

105 the base and top of the cloud, we can obtain the instantaneous water vapor flux into the cloud. If the temperature at the cloud top is lower than that at the cloud base, it can be considered that most of the water vapor entering the cloud has condensed. Assume that the water vapor condensation efficiency is $k$, and its value is less than 1 . We focus on the maximum possible condensate content, so $k$ is taken as 1 in this paper. The total hydrometer flux of the atmosphere per unit area in this period can be obtained by integrating it in a period of time, and the atmospheric column condensate can be expressed in formulas (2)

110 to (5):

$$
\begin{aligned}
& P_{w v}=P_{w v_{-} i n}-P_{w v_{-} \text {out }}, \\
& P_{w v_{-} i n}=\int_{t_{1}}^{t_{2}} S_{\text {base }}(T) v_{\text {base }}(t) d t \text {, } \\
& P_{w v_{-} \text {out }}=\int_{t_{1}}^{t_{2}} S_{\text {top }}(T) v_{\text {top }}(t) d t \text {, }
\end{aligned}
$$




$$
\begin{aligned}
& P_{\text {cong }}=k \cdot\left(P_{w v_{-} \text {in }}-P_{w v_{-} \text {out }}\right) \\
& =k \cdot \int_{t_{1}}^{t_{2}}\left(S_{\text {base }}(T) \cdot v_{\text {base }}(t)-S_{\text {top }}(T) \cdot v_{\text {top }}(t)\right) d t,
\end{aligned}
$$

115 Here, $p_{w v_{-} i n}$ is the input water vapor flux from the cloud base, $p_{w v_{-} o u t}$ is the output water vapor flux from the cloud top, and $p_{w v}$ is the water vapor flux remaining in the cloud layer in the period from $t_{1}$ to $t_{2} . S$ is the saturated water vapor density, which is related to the atmospheric temperature and the unit is $\mathrm{g} /\left(\mathrm{m}^{2} \cdot \mathrm{s}\right)$, and the subscripts "base" and "top" refer to cloud base and cloud top, respectively; $v$ is the vertical velocity of airflow, and the unit is $\mathrm{m} / \mathrm{s}$; the downward value is negative and the upward value is positive. $p_{\text {cong }}$ is the flux of hydrometeors converted from water vapor through condensation or sublimation in the period from $t_{1}$ to $t_{2}$.

Based on the ideal gas equation of state, the relationship between water vapor density $\rho_{v}$ and atmospheric temperature $T$ is in accordance with the following equation.

$\rho_{\mathrm{v}}=\frac{e}{R_{\mathrm{v}} T}=\frac{\varepsilon e}{R_{\mathrm{d}} T}$

Here, $\varepsilon$ is $0.622, R_{\mathrm{d}}$ is the constant of dry air and is $287.05 \mathrm{~J} / \mathrm{kg} \cdot \mathrm{K}, T$ is the absolute temperature in units of $\mathrm{K}$, and $e$ is the water vapor pressure $\left(\mathrm{kg} / \mathrm{m}^{3}\right)$. The saturated water vapor density can be derived from the saturated water vapor pressure at the same temperature. From the Tetens empirical formula, the saturated vapor pressure $e_{S}(T)$ of the liquid surface at temperature $T$ can be written as

$e_{s}(T)=610.78 \times \exp [17.2693882(T-273.16) /(T-35.86)]$,

If the temperature is below $0^{\circ} \mathrm{C}$, the value of $e_{S}(T)$ should be

$e_{s}(T)=611.2 \times \exp [17.67(T-273.15) /(T-29.65)]$.

The atmospheric column condensate is determined by the vertical velocity of airflow and the temperature difference at the cloud base and cloud top. If the detected cloud layer is very thick (if $>3 \mathrm{~km}$ ), the temperature at the cloud top will be much lower than that at the base, and the saturated water vapor density at the cloud top will be a very small value, so the water vapor term overflowing from the cloud top can be ignored. If the cloud layer is thin, such as a stratiform cloud, the water vapor from the cloud top cannot be ignored; otherwise, it will cause serious errors in the condensate calculation.

\section{Feasibility analysis of remote sensing observations for atmospheric column condensate}

If the temperature and vertical air velocity at the cloud base and cloud top can be obtained for a period of time, the condensate generated in the cloud layer during this period can be derived according to formula (6). It is difficult to continuously observe the vertical flow at the cloud base and cloud top. Observation instruments with high temporal and

140 spatial resolution are needed. Remote sensing instruments can realize continuous observation of atmospheric parameters. During the period of cloud change, the vertical air velocity near and inside a cloud deck varies rapidly and greatly. The 
vertical air velocity of stratiform clouds is very small, usually less than $1 \mathrm{~m} / \mathrm{s}$. The vertical air velocity at the base of the cumulonimbus cloud is relatively large and develops rapidly. The large dynamic detection range and high detection accuracy of remote sensing instruments are needed for detecting vertical airflow.

145 Lidar has proven to be a useful tool for atmospheric research. Today, lidar techniques for the remote sensing of atmospheric temperature profiles have reached the maturity necessary for routine observations (Behrendt et al., 2002; Wu et al., 2016). Lidar has been used for the evaluation of vertical air velocity near and inside a cloud deck (Lottman et al., 2001), and MWR was also used for the detection of vertical air motions (Kollias et al., 2002). The coherent Doppler lidar estimates the radial component of the velocity from the Doppler frequency or mean frequency of the backscattered laser field from atmospheric aerosol particles. The performance of coherent Doppler lidar is determined by the systematic and random errors of estimates for the mean frequency, which is related to the radial component of the velocity. The detection accuracy of coherent lidar for radial velocity can reach the order of $\mathrm{cm}$ (Frehlich et al. 1994). Lidar can detect vertical air motions at the cloud base (Lottman et al., 2001), but due to the weak penetration of clouds and fog by the laser, the speed of the thick cloud top cannot be measured by lidar. Compared with lidar, MWR has a stronger ability to detect clouds. MWR has the advantages of high sensitivity and strong penetration. It can measure the motion state inside the cloud. The cloud top height, cloud base height, and cloud dynamic parameters (such as the vertical velocity of air flow) were derived from the MWR. The small particle tracing method has been used to obtain the vertical air velocity in clouds for MWR. The detection resolution of MWR is on the order of centimeters (Jiafeng et al., 2017), and the detection accuracy is on the order of decimeters (Shupe et al., 2008). For stratiform clouds with low rising speeds, lidar should be used to detect the vertical air velocity at the cloud base. For convective clouds with higher rising speeds, MWR can also be used to detect the vertical air velocity at the cloud base.

Lidar (rotational Raman Lidar or high Spectral Resolution Lidar) and microwave radiometers have been developed to observe temperature and relative humidity $(\mathrm{RH})$ profiles. With rotational Raman lidar, temperature measurements can be carried out not only in the clear atmosphere but also in aerosol layers and optically thin clouds (Cooney, et al. 2016; Su et al., 2013). The temperature detection accuracy of rotating Raman lidar can be better than $1 \mathrm{~K}$ in low-altitude areas. For thick clouds, it is necessary to use a microwave radiometer to obtain the temperature profile in the cloud or at the cloud top. Compared with lidar, the range resolution and detection accuracy of microwave radiometers are low, but their maximum detection distance can reach $10 \mathrm{~km}$ and cover the top of most clouds.

Lidar, MWR and microwave radiometers need to be used together to realize the continuous observation of vertical air velocity and temperature at the cloud base and cloud top with high temporal and spatial resolution. The temperature at and below the cloud base can be obtained by lidar, and the value at the cloud top (if the cloud top height is less than $10 \mathrm{~km}$ ) is obtained by a microwave radiometer. The vertical velocity is measured by MWR. Fig. 2 shows the technical route of remote sensing observations for atmospheric column condensates. The temperature data from the sounding balloon are used to calibrate the results measured by Raman lidar and the microwave radiometer. 


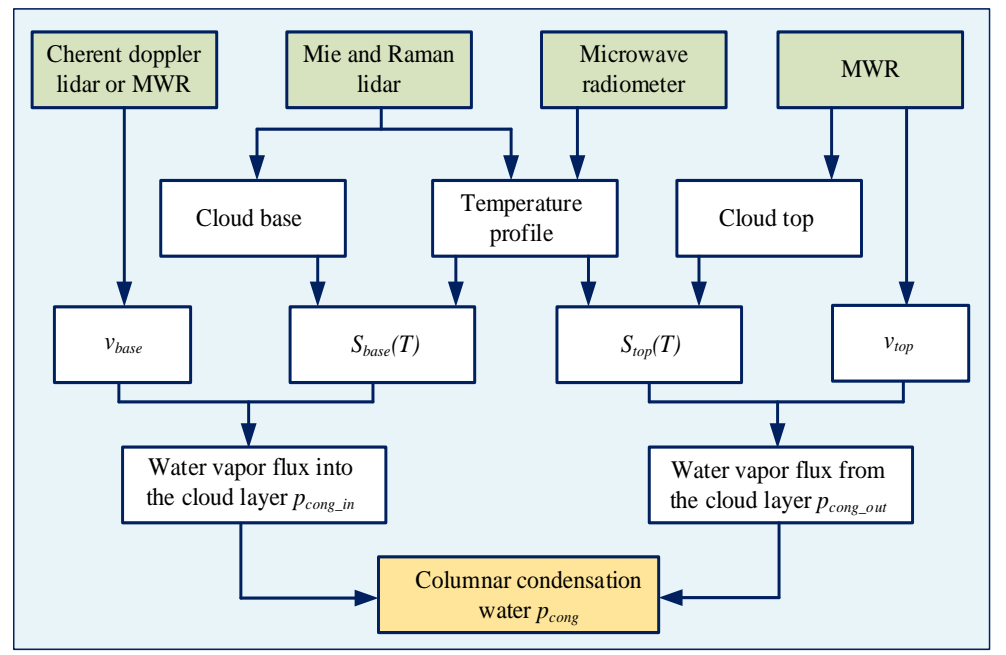

Figure 2: Technical route of remote sensing observations for atmospheric column condensate

Although lidar and MWR have the advantages of high spatial-temporal resolution, their detection accuracy for temperature and wind speed is affected by the system signal-to-noise ratio (SNR), which will affect the quantification accuracy of atmospheric column condensate. The determination of atmospheric column condensate depends on the water vapor flux into the cloud. According to the standard error propagation method, the uncertainty of the water vapor flux $\sigma_{p_{\mathrm{wv}}}$ depends on the 180 input and output flux errors $\sigma_{p_{\mathrm{w} \_ \text {_n }}}$ and $\sigma_{p_{\mathrm{w} \_ \text {out }}}$. They are determined by the uncertainty of the wind speed $\sigma_{v}$ and the saturated water vapor density $\sigma_{S}$ and yield

$$
\begin{gathered}
\sigma_{p_{\text {wv } \_ \text {in }}}=\sqrt{\sigma_{S_{\text {base }}}^{2} \cdot \overline{v_{\text {base }}}(\mathrm{t})^{2}+\sigma_{v_{\text {base }}}^{2} \cdot \bar{S}_{\text {base }}^{2}(T, t)} \\
\sigma_{p_{\text {wv } \_ \text {out }}}=\sqrt{\sigma_{S_{\text {top }}}^{2} \cdot \overline{v_{\text {top }}}(\mathrm{t})^{2}+\sigma_{v_{\text {top }}}^{2} \cdot \bar{S}_{\text {top }}^{2}(T, t)}
\end{gathered}
$$

The uncertainty of the saturated water vapor density depends on the temperature accuracy $\sigma_{T}$. The standard deviation can be obtained according to formulas (11) - (13):

$$
\begin{aligned}
& \sigma_{S}=\frac{\mathrm{d} S(T)}{\mathrm{d} T} \cdot \sigma_{T}, \\
& \frac{\mathrm{d} S(T)}{\mathrm{d} T}=\frac{2.17(T+273.15) \cdot \frac{\mathrm{d} e(T)}{\mathrm{d} T}+2.17 e(T)}{(T+273.15)^{2}}, \\
& \frac{\mathrm{d} e(T)}{\mathrm{d} T}=\frac{610.8 \times 17.27(2 T+237.3)}{(T+237.3)^{2}} \cdot \exp \left(\frac{17.27 T}{T+237.3}\right),
\end{aligned}
$$

The detection uncertainty of the instantaneous atmospheric column condensate changes with the cloud base temperature. The 190 temperature uncertainty of lidar at the cloud base is 0.5 to $1 \mathrm{~K}$, and the measurement uncertainty of wind speed is less than 
$0.1 \mathrm{~m} / \mathrm{s}$. Taking the spring of northern China as an example and assuming that the ground surface temperature is $15^{\circ} \mathrm{C}$ and the height of the cloud base is $3 \mathrm{~km}$, the temperature of the cloud base is approximately $-3^{\circ} \mathrm{C} \sim 4^{\circ} \mathrm{C}$. The standard deviation of the lidar for water vapor flux detection is approximately $0.7 \mathrm{~g} / \mathrm{m}^{2} \cdot \mathrm{s}$ according to formulas (9)-(13).

\section{Instrumentation}

195 The ground-based instruments used in this study are as follows: MWR, which provides reflectivity and Doppler spectrum data; microwave radiometer, which provides temperature profile; lidar, which can provide temperature, humidity profile, particle polarization characteristics, and more. These detection devices are installed in the Atmospheric Exploration Centre of the China Meteorological Administration. As shown in Fig. 3, the microwave radiometer and MWR are placed next to each other, approximately $50 \mathrm{~m}$ away from the lidar. The launch site of the sounding balloon is only $10 \mathrm{~m}$ from the lidar. The distance between these devices is approximately negligible compared with the observation target, so it provides a reliable research basis for subsequent joint observation and data inversion.

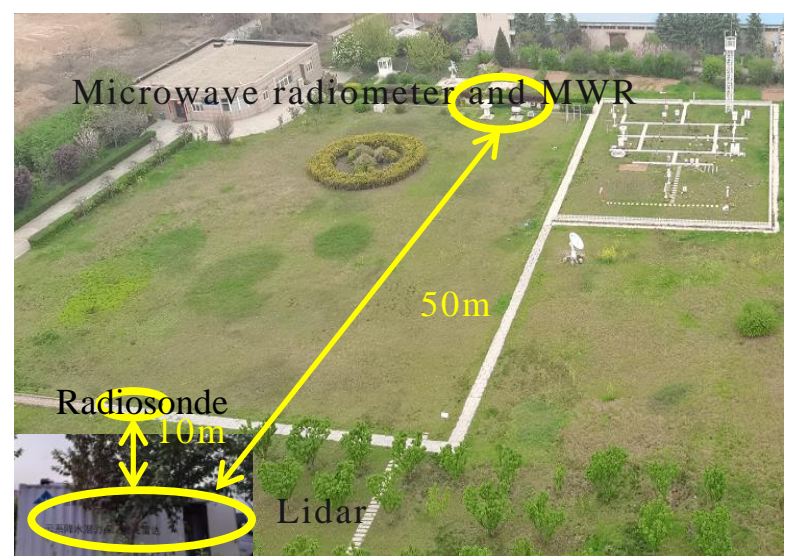

Figure 3: Installation of equipment at observation points

\section{Remote sensing technology of atmospheric temperature and vertical airflow velocity}

\subsection{Rotational Raman lidar for temperature profiles}

An ultraviolet rotational-vibrational-Mie Raman lidar system was established at Xi'an University of Technology $\left(34.233^{\circ} \mathrm{N}\right.$, $108.911^{\circ} \mathrm{E}$ ) in 2019 . The ultraviolet wavelength of $354.7 \mathrm{~nm}$ was chosen to enhance the vibrational-rotational Raman signals, as the intensity of molecular scattering is sensitive to the wavelength of the incident light. Lidar employs a Nd:YAG pulsed laser as the light source; its output energy at a frequency-tripled wavelength of $354.7 \mathrm{~nm}$ is $\sim 220 \mathrm{~mJ}$ at a $20 \mathrm{~Hz}$ repetition rate with a $9 \mathrm{~ns}$ FWHM pulse duration. The laser beam divergence is approximately $0.3 \mathrm{mrad}$. The receiver is constructed using a Cassegrain telescope with a diameter of $400 \mathrm{~mm}$ and a focal length of $2000 \mathrm{~mm}$. The collected lidar returns are coupled into a multimode fiber optic and guided into a high-efficiency spectroscopic box. All signals were detected by photomultiplier tubes (PMTs). Two pure rotational Raman (PRR) signals with different temperature dependences of the 
central wavelengths at $352.5 \mathrm{~nm}$ and $353.9 \mathrm{~nm}$ were selected to retrieve the atmospheric temperature profile. A set of dichroic mirrors (DMs) and narrow-band interference filters (IFs) with narrow angles of incidence were utilized to construct the high-efficiency 5-channel polychromator. Lidar can detect temperatures of $0.5 \sim 5 \mathrm{~km}$ under clear sky or thin cloud conditions. In addition to temperature, cloud base height is also important. An improved differential zero crossing method (Mao et al., 2010) is used for the retrieval of cloud base height.

Fig. 4 shows a set of range-squared correction signals (RSCS) (Fig. 4(a)) and temperature profile data retrieved from rotational Raman signals in cloudy weather detected by lidar. The height of the cloud base was approximately $4.5 \mathrm{~km}$. It also shows the radiosonde data and absolute deviation in the same period (Fig. 4(b)). Fig. 4(b) shows the temperature data of 0.7 $\mathrm{km}$ from the cloud base to the inside of the cloud retrieved, and there is a good match between the lidar and radiosonde data. The absolute deviation near the cloud base is less than $1 \mathrm{~K}$. Due to the influence of the overlap, the temperature below $1 \mathrm{~km}$ has a large deviation. Because this paper focuses on the temperature of the cloud base, the deviation of the base layer is not considered. Fig. 4 (c) shows the temperature statistical error calculated according to the signal-to-noise ratio (SNR) of the lidar. The temperature uncertainty near the cloud base is less than $0.5 \mathrm{~K}$. To further estimate the reliability of Raman lidar, we used 50 sets of temperature profiles to perform the regression analysis between the lidar and the radiosonde data. As shown in Fig. 5, the correlation coefficient reached 0.98 below a height of 5 km, and the root mean square error (RMSE) was $1.2 \mathrm{~K}$.
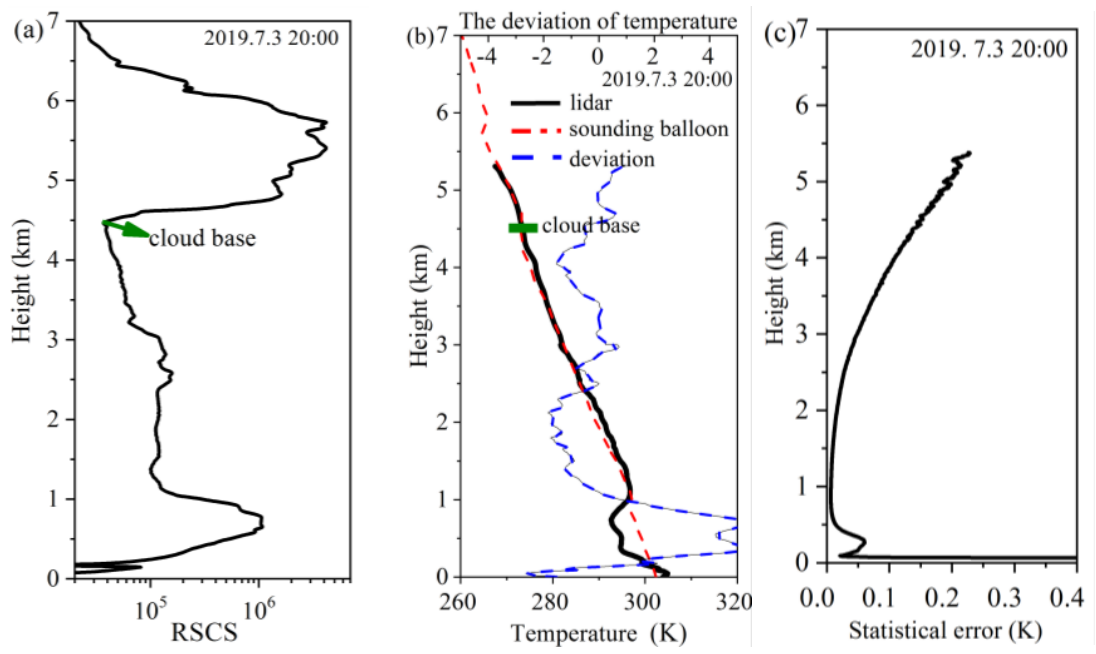

Figure 4: Mie-scattering signal (a), temperature profile (b) and statistical error (c) detected by rotational Raman lidar 


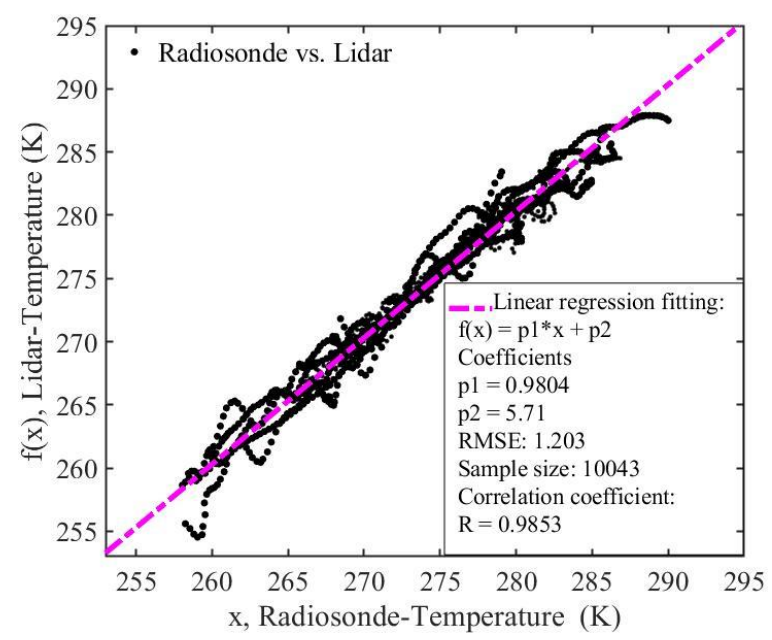

Figure 5: Regression analysis between the Raman lidar and radiosonde data and the root-mean-square of temperature

\subsection{MWR for vertical airflow velocity}

235 Vertically pointing $35 \mathrm{GHz}$ MWR (Williams et al., 2016) is often used in cloud research. The size of cloud particles is related to the natural falling velocity of cloud particles. The radial velocity measured by vertically pointing MWR includes the falling velocity of cloud particles in a static atmosphere and atmospheric vertical velocity. For micron-scale cloud particles, the natural falling speed is very small (within $2 \mathrm{~cm} / \mathrm{s}$ ), and the vertical motion speed of air can be $1 \sim 2$ orders of magnitude larger than that of cloud particles (Kollias et al., 2001; Kollias et al.,2011).

240 Both vertical air motions and particle fall speeds contribute to the Doppler velocities measured by cloud radars (Gossard et al., 1996). Moreover, the spectrum of radar Doppler velocities is influenced by turbulence within the radar volume. Thus, the derivation of vertical air motions from vertically oriented cloud radar measurements is only possible under specific sets of conditions. First, with explicit knowledge of the cloud particle size distribution, a quiet-air spectrum of particle fall speeds can be computed that can then be compared to the measured radar Doppler spectrum to provide an estimate of the vertical air motion (O'Connor et al., 2005). Quiet-air Doppler spectra can also be determined by deconvolving the fall speed and turbulent contributions to the measured radar Doppler spectrum. Such methods rely on assumptions about the shape of the particle size distribution and the particle size-fall speed relationship (Gossard 1994; Gossard et al., 1996) and/or computationally intensive inversion processes (Babb et al. 1999). If only the vertical velocity is desired, a much simpler retrieval can be applied that only requires prior knowledge about the presence of small particles, such as liquid water cloud droplets, in the radar volume that are assumed to be tracers of clear-air motions (Gossard 1994; Kollias et al., 2001; Shupe et al., 2008). Here, we exploit this last condition to derive vertical air velocities from cloud radar measurements. As shown by $\mathrm{W}_{\text {air }}$ in Fig. 6, it is the vertical velocity of the atmosphere. 


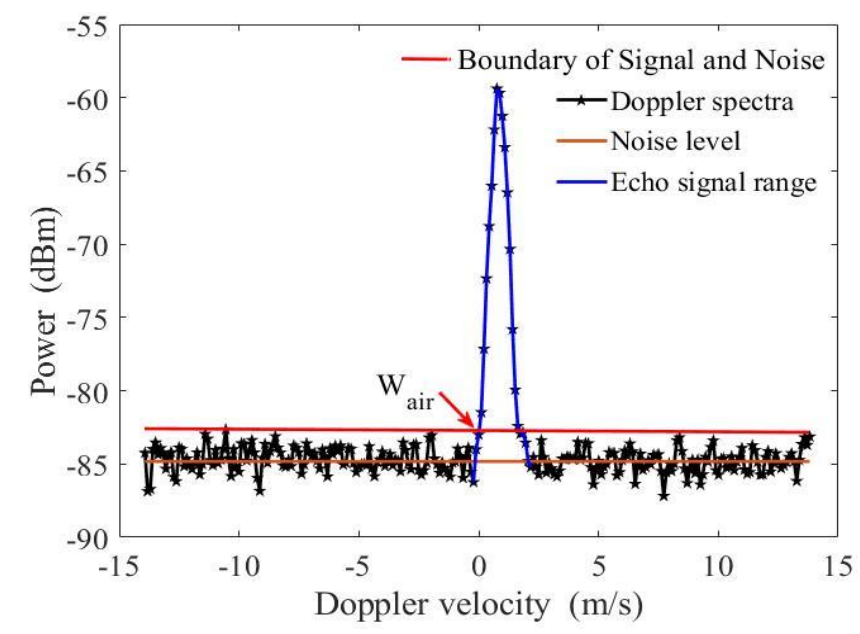

Figure 6: Schematic diagram of power spectrum data processing

255 Precise wind field measurement under rainy conditions is a challenge due to the presence of interfering signals from raindrop reflections (Kollias et al., 2014). Precipitation particles often appear in cloud layers, so it is very important to distinguish the falling velocity of raindrops and the vertical airflow velocity. Under rainy conditions, the received backscattering signal contains two components, the cloud particle signal and the precipitation particle signal. Two peaks of the Doppler power spectrum can be observed if the wind and rainfall velocities are different. The vertical airflow and raindrop falling velocity can be distinguished according to the difference in their power spectra.

\section{A case study for observation of atmospheric column condensate}

\subsection{Joint detection of clouds by remote sensing instruments}

Stratiform clouds were observed by lidar and MWR during the period from June 8, 2021, to June 9, 2021. During this period, a weak rainfall process occurred, and the clouds dissipated later. Fig. 7(a) shows the RSCS at $1064 \mathrm{~nm}$ from the lidar, and 265 Fig. 7(b) shows the results of the MWR. 


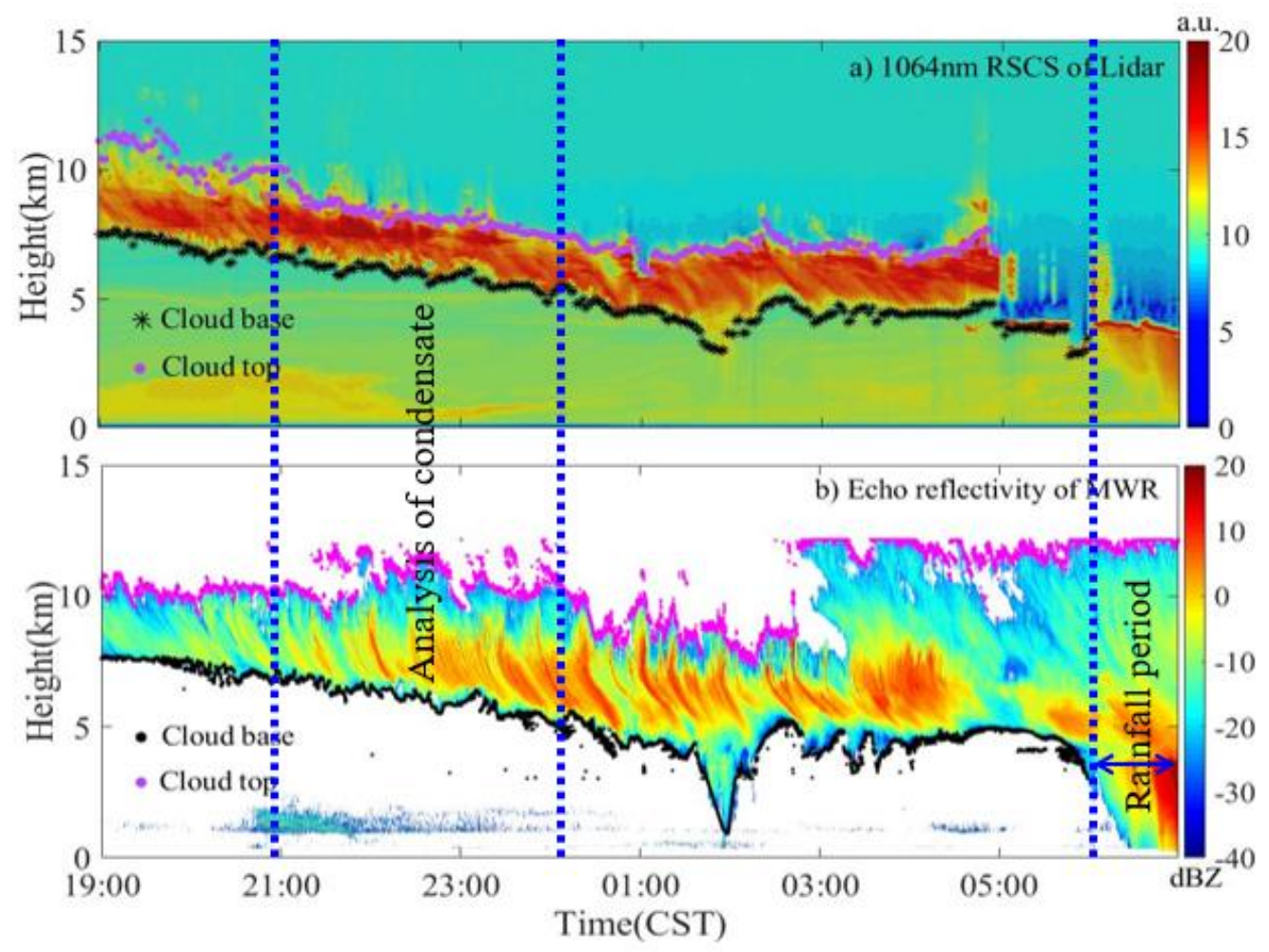

Figure 7: Time-height-indicator (THI) plots of the range-squared-corrected signal (RSCS) observed by lidar at 1064 nm; (b) time-

According to Fig. 7, the cloud layer was detected simultaneously by lidar and MWR, and the cloud base height decreased from $\sim 8 \mathrm{~km}$ to $\sim 0.5 \mathrm{~km}$ during the observation period. The cloud base height was maintained at approximately $5 \mathrm{~km}$ from 02:00 to 05:00 on June 9, 2021, while the cloud thickness increased. It was rain on June 9, 2021. Lidar could not effectively detect rainfall; however, cloud changes during rainfall were effectively observed by MWR. The penetration ability of MWR is stronger than that of lidar. The value distribution equalization (VED) (Zhao et al., 2014) method is used to calculate the cloud base height change detected by the lidar (black mark in Fig. 7 (a)). Based on the MWR detection mechanism (MATTHEW et al., 2007), the cloud base and cloud top of the MWR are derived (Fig. 7 (b)). Black points represent the cloud base, and flat red points represent the cloud top. Because the penetration ability of lidar is not as good as that of MWR, the height of the cloud top should be subject to the detection result of MWR. The cloud base height values detected by the two devices are shown in Fig. 8. The cloud base height and its change trend detected by the two devices have a high degree of coincidence in the observation time period (before rainfall). Their correlation analysis is carried out. The results are shown in Fig. 8, and the correlation is 0.9256 . It is verified again that the two devices have a certain consistency on the macro parameters of clouds in the process of joint observation. 

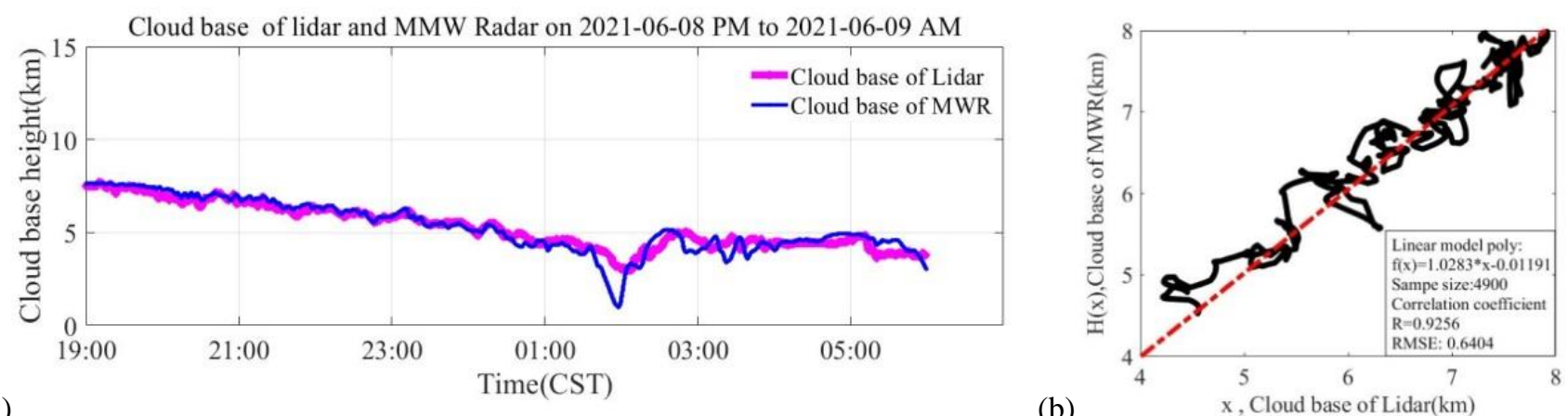

(a)

(b)

$\mathrm{x}$, Cloud base of Lidar $(\mathrm{km})$

Figure 8: Cloud base heights detected by lidar and MWR. (a) Time series of cloud base height, (b) correlation analysis of cloud base height

During the period of observation, the cloud phase state also changes with the thickness and height of the cloud. Through the discrimination and identification of the cloud phase state during the observation process (Shupe et al., 2008), it is determined that the cloud phase state is a mixed phase state from 21:00 to 23:00 on June 8, 2021, and the state is relatively stable. The base of the cloud is a liquid water area. In this detection period, the small particle tracing method can be used to accurately obtain the atmospheric vertical velocity $\left(\mathrm{W}_{\text {air }}\right)$, which can improve the accuracy of the condensate determination.

\subsection{Temperature and vertical wind velocity at the cloud base and top}

The temperature information below the cloud was retrieved according to the rotational Raman signal of lidar. Combined with the cloud base position, the time series of cloud base temperature was obtained. The temperature of the cloud top cannot be detected by lidar. The microwave radiometer data were chosen as a supplement for temperature. The results of the microwave radiometer were checked by sounding balloon data, and the temperature deviation of the microwave radiometer was less than $2 \mathrm{~K}$. The time series of cloud top temperatures was also derived. The temperature results at the cloud base and top (2021-06-08 21:00 23:00) are shown in Fig. 9.

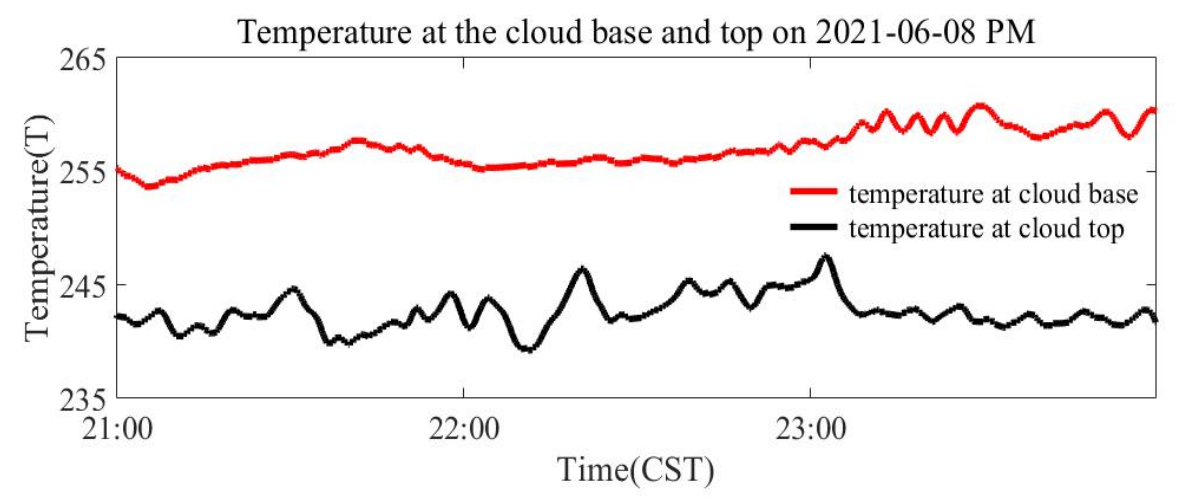

The power spectrum data of the MWR from 21:00 to 23:00 on June 8, 2021 by analyzing, the air vertical motion velocity at the cloud base and cloud top (positive upward and negative downward) was obtained by the small particle tracing method 
described above (subsection 6.2). The results are shown in Fig. 10. The calculated atmospheric vertical velocity value is relatively consistent with MATTHEW (MATTHEW et al., 2007). Fig. 10 shows that there was mainly an updraft at the cloud base, and the updraft and downdraft at the cloud top were staggered, but mainly the downdraft, which is also in line with the phenomenon of cloud top height decline.

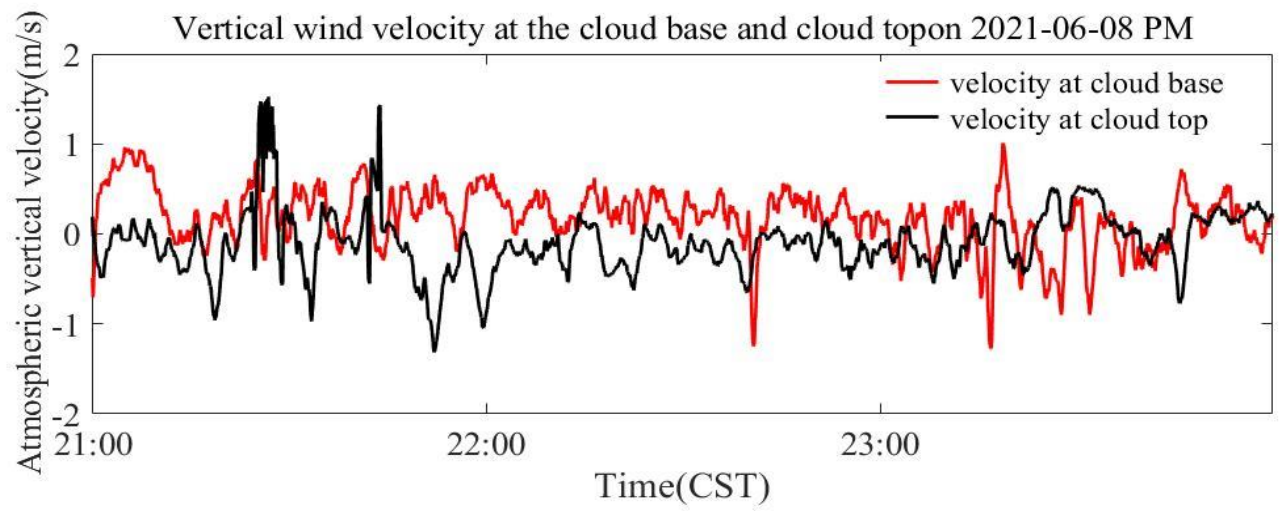

Figure 10: Time series of vertical wind velocity at the cloud base and cloud top

\subsection{Results of atmospheric column condensate}

The water vapor flux was calculated according to Eq. (2)-(8). From Fig. 10, the cloud top temperature is approximately $12^{\circ} \mathrm{C} \sim 15^{\circ} \mathrm{C}$ lower than the cloud base temperature; therefore, it can be considered that the water vapor remaining in the cloud can condense to a large extent. The rising air at the cloud top brings water vapor out, reducing the content of condensate in the cloud. Fig. 11 shows the water vapor flux through the cloud base and cloud top. There is both water vapor entering from the cloud base and water vapor leaving from the cloud top in this observed case. The water vapor flux entering the cloud base minus the water vapor flux leaving the cloud top is the value remaining in the cloud layer, which can be considered the maximum possible condensate flux. If the water vapor flux is positive, the cloud is constantly generating and developing; if it is negative, the cloud is dissipating. Fig. 12 shows the instantaneous water vapor flux remaining in the cloud layers.

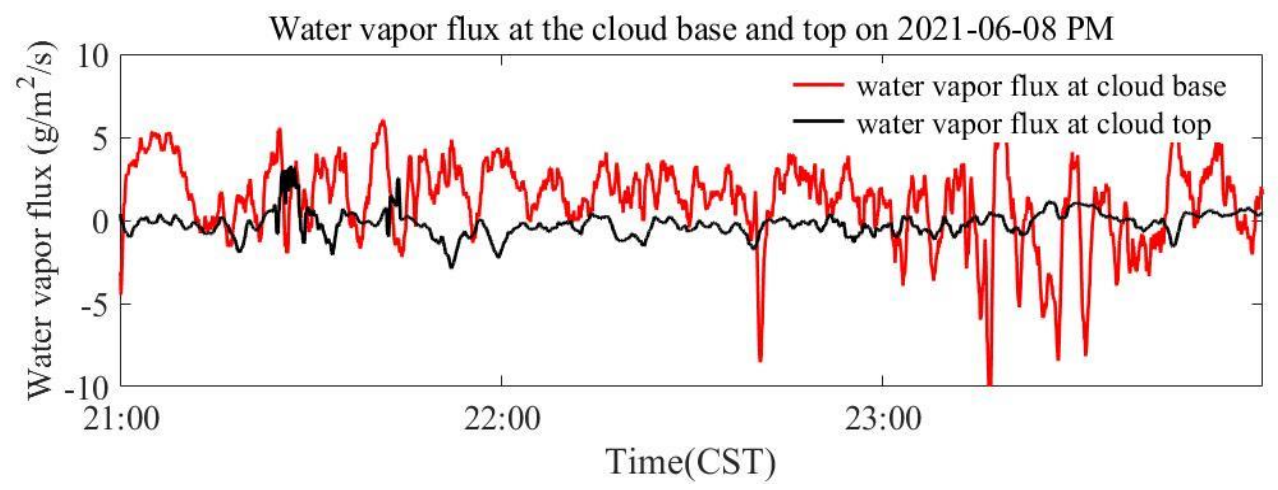

Figure 11: Time series of water vapor flux at the cloud base and top 


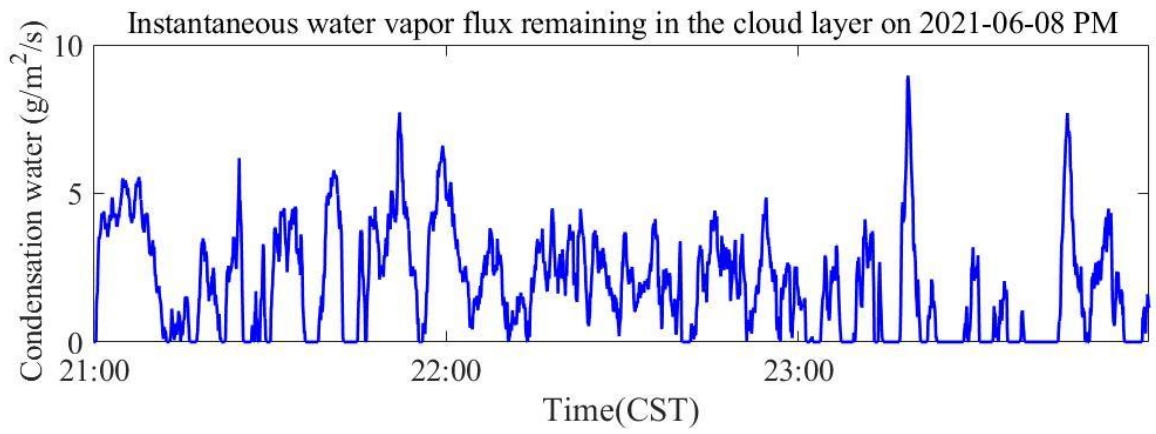

Figure 12: The instantaneous water vapor flux remaining in the cloud layer

The atmospheric column condensate can be obtained by integrating the instantaneous water vapor flux. Fig. 13 shows the hourly condensate during the observation period. From Fig. 11, the updraft at the cloud top was very small, so it could be considered that the water vapor entering the cloud basically stayed in the cloud. According to Eq. (5), the condensate in the period from 21:00 to 23:00 was integrated, and it could be seen that the total amount of the maximum possible condensate in the atmosphere in this period was approximately $88.2 \mathrm{~g}(2.94 \mathrm{~mm})$. The water condensed in this period provided resources for the incoming rainfall at 06:00 a.m. on June 9, 2021. Therefore, the detection and derived results are in good agreement with the changes in weather and clouds on that day.

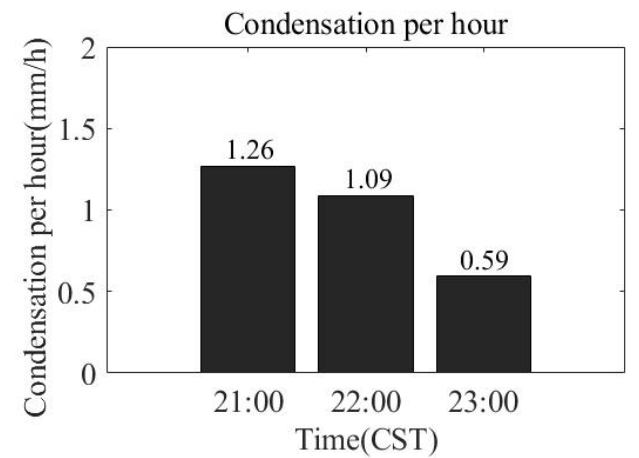

Figure 13: Hourly condensate during the observation period

\section{Results and discussions}

It is very difficult to directly measure atmospheric column condensate. According to the characteristics of different remote

sensing equipment, the condensate content can be deduced by measuring the meteorological parameters near and inside a cloud deck. This paper presents a remote sensing method for detecting atmospheric column condensates by using lidar, MWR and microwave radiometry. The detailed detection scheme and data calculation method are given. A precipitation process was observed, and atmospheric column condensate was obtained by active and passive instruments. In this method, it is considered that the water vapor remaining in the cloud can condense, which is an ideal assumption. In fact, a part of the 
340 water vapor entering the cloud evaporates or exits the cloud boundary, which will lead to the reduction of the condensate. These effects are not considered in this paper. We provide the maximum possible atmospheric column condensate. Therefore, the derived condensate should be larger than the actual value. Nevertheless, the method proposed in this paper is still valuable. The method proposed in this paper only uses the observation data of one point to represent the results of the whole region, which is reasonable for horizontally uniform stratiform clouds. For clouds with strong convection, it is more effective

345 to use multipoint network observation data.

Stratiform clouds often appear in northern China and are the main targets of artificial weather modification. The vertical airflow velocity under stratiform clouds is small and should be obtained by using high-precision detection equipment. Lidar is an effective instrument to obtain high-precision wind speed and temperature under clouds. Precipitation is a complex weather system that can be divided into two categories: convective precipitation and systematic precipitation. Convective

350 precipitation generally covers a small range. The horizontal scale of convective clouds is on the order of $100 \mathrm{~km}$, and the water vapor supply is mainly provided by the rise of air on the ground. The updraft before precipitation can be measured by MWR or lidar to obtain the "potential precipitation", that is, the total amount of cloud water in a certain area and a certain period of time. After the beginning of precipitation, if the precipitation is very weak, lidar can also detect the temperature and updraft in the precipitation area, but when there is heavy precipitation, lidar will not be able to obtain effective data.

355 Systematic precipitation is complex, and a multilayer cloud structure will appear. Lidar usually cannot penetrate multilayer clouds, and it is only suitable for the detection of the base of single-layer clouds. For multilayer clouds, it is necessary to use MWR to detect the vertical air velocity in multilayer clouds and a microwave radiometer to detect the temperature in clouds. The assessment of CWR in an area requires long-term statistical results, which require long-term continuous remote sensing observations. This paper provides a detection method for atmospheric column condensates and a scientific observation case.

360 More cloud observation results will be carried out in the future for CWR assessment.

\section{Data availability}

Data and code related to this article are available upon request to the corresponding author.

\section{Author contributions}

The conceptualization was performed by HD and DH. The methodology and investigation were completed by HD, YY, QY,

365 WX, JW and SL. HD drafted the original manuscript, which was reviewed and edited by YY, HD. YW contributed to significantly improve the manuscript. Supervision was the responsibility of DH and LZ, and project administration by DH.

\section{Competing interests}

The authors declare that they have no conflict of interest. 
https://doi.org/10.5194/amt-2021-397

Preprint. Discussion started: 18 February 2022

(c) Author(s) 2022. CC BY 4.0 License.

(c) (i)

\author{
Atmospheric \\ Measurement \\ Techniques \\ Discussions
}

\section{Acknowledgements}

370 We are very grateful to Jietai Mao (Peking University) for providing the useful suggestions and interpretation and definition of atmospheres cloud-water resources for this manuscript, and we are thankful to the Xi'an Meteorological Administration of China for supporting the data collection and archiving.

\section{Financial support}

This research has been supported by National Natural Science Foundation of China (NSFC): 41627807. National Natural

375 Science Foundation of China (NSFC): 42130612.

\section{References}

Behrendt, A. \& Nakamura, T.: Calculation of the calibration constant of polarization lidar and its dependency on atmospheric temperature. Opt Express., 10(16), 805-17, doi:10.1364/OE.10.000805,2002.

380 Cooney, \& John.: Measurement of atmospheric temperature profiles by raman backscatter. J Appl Meteorol Clim., 11(1), 108-112, doi:10.1175/1520-0450 (1972)011<0108:moatpb>2.0.co;2,1972.

Edward P. Luke, Pavlos Kollias.: Separating Cloud and Drizzle Radar Moments during Precipitation Onset Using Doppler Spectra. J Atmos Ocean Tech., 30,1656-1671, doi:10.1175/JTECH-D-11-00195.1, 2012.

Frehlich, R., Hannon, S. M., \& Henderson, S. W.: Performance of a 2-m coherent doppler lidar for wind measurements. J.atomos.oceanic Technol.,11(11), 1517-1528. doi:10.1175/1520-0426(1994)0112.0.CO;2,1994.

Gossard, E. E., Snider, J. B., Clothiaux, E. E., Martner, B., \& Frisch, A. S.: The potential of 8-mm radars for remotely 390 sensing cloud drop size distributions. J Atmos Ocean Tech.,14(1),76-87,doi:10.1175/15200426(1997)014<0076:TPOMRF>2.0.CO;2,1996.

Gossard, E. E.: Measurement of cloud droplet size spectra by doppler radar. J Atmos Ocean Tech., 11(3), 712-726, doi:10.1175/1520-0426(1994)0112.0.CO;2,1994.

Gu, X., \& Bing, Z.: Vapor sink and latent heat of condensation in the atmosphere and the parameterization of cumulus convection. Acta Meteorol Sin., 64(6), 790-795,doi:10.1016/S1872-2032(06)60022-X,2006. 
Hengchi, L., Dezhen, J., Chong, W., \& Zhilai, S.: An airborne microwave radiometer and measurements of cloud liquid water. Chinese Sci Bull., 48(0z2), 82-87, doi:10.1360/03wd0462,2003.

Jalihal, C., Srinivasan, J., \& Chakraborty, A.: Modulation of indian monsoon by water vapor and cloud feedback over the past 22,000 years. Nat. Commun., 10:5701, doi:10.1038/s41467-019-13754-6,2019.

Jiafeng, Z., Liping, L., Keyun, Z., Jingya, W., \& Binyun, W.: A method for retrieving vertical air velocities in convective clouds over the tibetan plateau from tipex-iii cloud radar doppler spectra. Remote Sens.,9, 964,2-17, doi:10.3390/rs9090964,2017.

Kollias, P., Albrecht, B. A., \& F Marks.: Why mie? accurate observations of vertical air velocities and raindrops using a cloud radar. Bull.amer.meteor.soc., 83(10), 1471-1483, doi:10.1175/BAMS-83-10-1471,2002.

Kollias, P., Albrecht, B. A., Lhermitte, R., \& Savtchenko, A.: Radar observations of updrafts, downdrafts, and turbulence in fair-weather cumuli. J Atmos Sci.,58(13),1750-1766, doi:10.1175/1520-0469(2001)0582.0.CO;2,2001.

Kollias, P., Jasmine Rémillard, Luke, E., \& Szyrmer, W.: Cloud radar doppler spectra in drizzling stratiform clouds: 1. 415 forward modeling and remote sensing applications. J Geophys Res-Atmos., 116 ,D13201,doi:10.1029/2010JD015237,2011.

Liu, F., \& Yi, F.: Spectrally resolved raman lidar measurements of gaseous and liquid water in the atmosphere. Appl Opt., 52(28),6884-6895, doi:10.1364/AO.52.006884,2013.

420 Liu, W. Q., Chen, Z. Y., Liu, J. G., \& Xie, P. H.: Stereoscopic monitoring technology and applications for the atmospheric environment in China. Chinese Sci Bull., 61(30), 3196-3207, doi:10.1360/N972016-00394,2016.

Lottman, B. T., \& Frehlich, R. G.: Evaluation of vertical winds near and inside a cloud deck using coherent doppler lidar. J Atmos Ocean Tech., 18(8), 1377-1386, doi:10.1175/1520-0426 (2001)0182.0.CO;2,2001.

Mao F, Gong, W., Li, J., \& Zhang, J.: Cloud detection and parameter retrieval based on improved differential zero-crossing method for mie lidar. Acta Optica Sinica.,30(11),3097-3102, doi:10.3788/AOS20103011.3097,2010.

MATTHEW D. SHUPE, PAVLOS KOLLIAS, P. OLA G. PERSSON, GREG M. MCFARQUHAR.: Vertical Motions in 430 Arctic Mixed-Phase Stratiform Clouds. J Atmos Sci., 65,1304-1322, doi:10.1175/2007JAS2479.1,2007. 
O'Connor, E. J., Hogan, R. J., \& Illingworth, A. J.: Retrieving stratocumulus drizzle parameters using doppler radar and lidar. J Appl Meteorol Clim., 44,14-27, doi:10.1175/JAM-2181.1,2005.

Pavlos, Kollias, Wanda, Szyrmer, Jasmine, \& Rémillard.: Cloud radar doppler spectra in drizzling stratiform clouds: 2. observations and microphysical modeling of drizzle evolution. J Geophys Res., 116(D13), doi:10.1029/2010jd015238,2011.

Shupe, M. D., Kollias, P., Poellot, M., \& Eloranta, E.: On deriving vertical air motions from cloud radar doppler spectra. J Atmos Ocean Tech., 25(4), 547-557, doi:10.1175/2007JTECHA1007.1,2008.

440 Si, A., Ts, A., Ho, B., Ks, B., Sk, B., \& Mf, C., et al.: Large-and-sparse-particle clouds (lsc): clouds that are subvisible for space-borne lidar and observable for space-borne cloud radar. Polar Sci., 21, 117-123, doi:10.1016/j.polar.2019.05.003,2019.

Su T., \& Feng G.L.: The characteristics of the summer atmospheric water cycle over China and comparison of ERA-Interim and MERRA reanalysis.Acta Phys Sin., 63(24),493-505,doi:10.7498/aps.63.249201,2014.

Su, J., Mccormick, M. P., Wu, Y., Lee, R. B., Lei, L., \& Liu, Z.: Cloud temperature measurement using rotational raman lidar. J Quant Spectrosc Ra., 125, 45-50, doi:10.1016/j.jqsrt.2013.04.007,2013.

Williams, C. R., Beauchamp, R. M., \& Chandrasekar, V.: Vertical air motions and raindrop size distributions estimated using mean doppler velocity difference from 3- and 35-ghz vertically pointing radars. IEEE T Geosci Remote.,54(10),113,doi:10.1109/TGRS.2016.2580526,2016.

Wu, D., Wang, Z., Wechsler, P., Mahon, N., \& Heesen, B.: Airborne compact rotational raman lidar for temperature measurement. Opt Express., 24(18), A1210- A1223, doi:10.1364/OE.24.0A1210,2016.

Yao J.Q., Chen Y.N., Zhao Y., Guan X.F., Mao W.Y., \& Yang L.M.: Climatic and associated atmospheric water cycle changes over the Xinjiang, China. J Hydrol.,585, 124823, doi:10.1016/j.jhydrol.2020.124823,2020.

Yoshiaki, S., MD Yamanaka, Hiroyuki, H., Akira, W., Hiroshi, U., \& Yasuyuki, M.: Hierarchical structures of vertical velocity variations and precipitating clouds near the baiu frontal cyclone center observed by the mu and meteorological radars. J Meteorol Soc Jpn.,75(2), 569-596, doi:10.2151/jmsj1965.75.2_569,2009.

Zhao, C., Wang, Y., Wang, Q., Li, Z., Wang, Z., \& Liu, D.: A new cloud and aerosol layer detection method based on micropulse lidar measurements.J. Geophys. Res. Atmos., 119,6788-6802, doi:10.1002/2014JD021760,2014. 
https://doi.org/10.5194/amt-2021-397

Preprint. Discussion started: 18 February 2022

(c) Author(s) 2022. CC BY 4.0 License.

(c) (i)

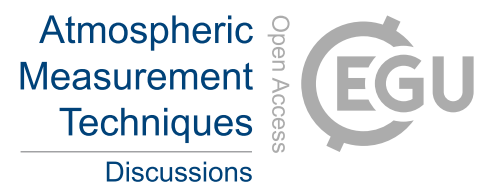

Zhou Y. Q., Cai M., OU J.I., Cai Z. X., \& Shi A.L.: Correlation between cloud characteristic parameters and precipitation. J Atmos Sci., 34(6):641-652, doi:10.3969/j.issn.1674-7097.2011.06.002,2011.

Zhou, L., Liu, Q., Liu, D., Xie, L., Qi, L., \& Liu, X.: Validation of modis liquid water path for oceanic nonraining warm clouds: implications on the vertical profile of cloud water content. J Geophys Res-Atmos., 121(9), 4855-4876, 470 doi:10.1002/2015JD024499,2016.

Zhou, Y., Cai, M., Tan, C., Mao, J., \& Zhijin, H. U.: Quantifying the cloud water resource: basic concepts and characteristics. J Meteorol Res-Prc.,34(6), 1242-1255, doi:10.1007/s13351-020-9125-7,2020. 